\title{
The interplay between transport and metabolism in fungal itaconic acid production
}

Hamed Hosseinpour Tehrani ${ }^{1 \ddagger}$ Elena Geiser $^{1,4 \ddagger}$ Meike Engel $^{1}$, Sandra K. Hartmann ${ }^{1}$, Abeer H. Hossain ${ }^{2}$, Peter J. Punt ${ }^{2}$, Lars M Blank ${ }^{1} \&$ Nick Wierckx*1,3

These authors contributed equally to this manuscript

*to whom correspondence should be addressed

${ }^{1}$ iAMB - Institute of Applied Microbiology, ABBt - Aachen Biology and Biotechnology, RWTH Aachen University, Worringerweg 1, 52074 Aachen, Germany

${ }^{2}$ Dutch DNA Biotech B.V, Utrechtseweg 48, 3704 HE, Zeist, The Netherlands

${ }^{3}$ Institute of Bio- and Geosciences IBG-1: Biotechnology, Forschungszentrum Jülich, 52425 Jülich, Germany

${ }^{4}$ BioSC, c/o Forschungszentrum Jülich, 52425 Jülich, Germany

Address for correspondence:

Prof. Dr. Nick Wierckx

Phone: $\quad$ +4924616185247

Fax: $\quad$ +492461612710

email: $\quad$ n.wierckx@fz-juelich.de

\section{Author copy}

This is the pre-peer reviewed version of the following article: Hosseinpour Tehrani, H., E. Geiser, M. Engel, S.K. Hartmann, A. Hossain, P. Punt, L.M. Blank, N. Wierckx. 2019. The interplay between transport and metabolism in fungal itaconic acid production. Fungal Genet. Biol. 25:45-52, which has been published in final form at https://doi.org/10.1016/j.fgb.2019.01.011.

\begin{abstract}
Besides enzymatic conversions, many eukaryotic metabolic pathways also involve transport proteins that shuttle molecules between subcellular compartments, or into the extracellular space. Fungal itaconate production involves two such transport steps, involving an itaconate transport protein (Itp), and a mitochondrial tricarboxylate transporter (Mtt). The filamentous actinomycete Aspergillus terreus and the unicellular basidiomycete Ustilago maydis both produce itaconate, but do so via very different molecular pathways, and under very different cultivation conditions. In contrast, the transport proteins of these two strains are assumed to have a similar function. This study aims to investigate the roles of both the extracellular and mitochondrial transporters from these two organisms by expressing them in the corresponding $U$. maydis knockouts and monitoring the extracellular product concentrations. Both transporters from A. terreus complemented their corresponding $U$. maydis knockouts in mediating itaconate production. Surprisingly, complementation with Mtt of A. terreus (At_MfsA) led to a partial switch from itaconate to $(S)$-2-hydroxyparaconate secretion. Apparently, the export protein from A. terreus has a higher affinity for (S)-2-hydroxyparaconate than for itaconate, even though this species is classically regarded as
\end{abstract}


an itaconate producer. Complementation with At_MttA increased itaconate production by 2.3-fold compared to complementation with Um-Mtt1, indicating that the mitochondrial carrier from A. terreus supports a higher metabolic flux of itaconic acid precursors than its $U$. maydis counterpart. The biochemical implications of these differences are discussed in the context of the biotechnological application in $U$. maydis and A. terreus for the production of itaconate and (S)-2-hydroxyparaconate.

\section{Keywords}

Itaconate

(S)-2-hydroxyparaconate

Ustilago maydis

Aspergillus terreus

Transporter

Metabolism 


\section{Introduction}

Itaconic acid can be used as a bio-based building block for the synthesis of a large number of chemicals and polymers. In 2004, the U.S. Department of Energy declared itaconic acid as one of the top 12 bio-based platform chemicals with high biotechnology potential (Werpy, 2004). Nowadays, it is mainly used in the production of synthetic fibers, for coatings, thickeners, anti-scaling agents in water treatments, and in the pharmaceutical sector (Geilen et al., 2010; Klement \& Buchs, 2013; Okabe et al., 2009; Weastra, 2014; Willke \& Vorlop, 2001). It's role as central mammalian immunoregulator has recently been reported opening novel uses, e.g., in the treatment for autoimmune conditions (Bambouskova et al., 2018). Itaconate can also be converted by fungi into the chiral lactone (S)-2-hydroxyparaconate (Geiser et al., 2016; Stodola et al., 1945). The functional groups within this product can be further manipulated in many directions to access a multitude of high-value and novel fine chiral chemicals with a wide range of potential applications ranging from medicine to pesticides to quantum computing (Brandt et al., 2017; Mori \& Fukamatsu, 1992). The unicellular basidiomycete Ustilago maydis and the filamentous actinomycete Aspergillus terreus both naturally produce itaconate (Fig. 1). In both organisms, the genes enabling itaconate biosynthesis are clustered and co-regulated (Geiser et al., 2015; Li et al., 2011). The biosynthetic pathway starts with the transport of cis-aconitate from the mitochondria to the cytosol by a mitochondrial tricarboxylate transporter, encoded by Um_mttl or At_mttA (Geiser et al., 2016; Geiser et al., 2015; Steiger et al., 2016). Both transporters are assumed to do so by antiport exchange with cytosolic malate (Jaklitsch et al., 1991). In $U$. maydis MB215, this transport poses the rate-limiting step in itaconate production, since overexpression of $m t t 1$ leads to a strong increase in itaconate production (Geiser et al., 2015). In contrast, overexpression of $m t t A$ has no significant impact on itaconate production by A. terreus LYT10 (Huang et al., 2014), although heterologous overexpression of $m t t A$, along with $\operatorname{cadA}$, did have a positive impact on itaconate production in Aspergillus niger (Li et al., 2011; van der Straat et al., 2014). In A. terreus, the cytoplasmic cis-aconitate is converted directly to itaconate by a cytosolic cis-aconitate decarboxylase (cadA) (Bonnarme et al., 1995; Dwiarti et al., 2002; Kanamasa et al., 2008). In contrast, U. maydis first isomerizes cis-aconitate to trans-aconitate by a cytosolic aconitate- $\delta$-isomerase (Adi1). This trans-aconitate is subsequently decarboxylated to itaconate by a trans-aconitate decarboxylase (Tad1) (Geiser et al., 2015).

Recently, Geiser et al. (2016) demonstrated that in U. maydis, itaconate is further converted to (S)-2-hydroxyparaconate by an itaconate P450 monooxygenase (Cyp3). The latter is the lactone of L-itatartarate, which is also found in the supernatants of $U$. maydis (Guevarra \& Tabuchi, 1990a; Guevarra \& Tabuchi, 1990b). Some A. terreus strains have also been reported to produce these products (Guevarra \& Tabuchi, 1990a; Jadwiga \& Diana, 1974), likely through a similar P450 enzyme encoded by the cypC gene directly adjacent to the itaconate gene cluster (Geiser et al., 2016; Steiger et al., 2013). However, (S)-2-hydroxyparaconate production is not reported for strains such as A. terreus NRRL 1960 or A. terreus DSM 23081 (Krull et al., 2017; Kuenz et al., 2012), possibly because these strains were selected by screening for high itaconate production leading to the selection of a defect in $c y p C$ expression. Both $U$. maydis and $A$. terreus possess a major facilitator superfamily transporter, encoded by Um_itpl and At_mfsA, which are assumed to be itaconate exporters (Geiser et al., 2016; Hossain et al., 2016; Li et al., 2011). Overexpression of $m f_{s} A$ in A. terreus and A. niger led to an increase in itaconate production (Huang et al., 2014; Li et al., 2011), while itpl overexpression in $U$. maydis did not increase production (Geiser et al., 2016). However, in light of the fact that both itaconate and $(S)$-2-hydroxyparaconate are produced via the same pathway, and that the gene clusters of both organisms only contain one gene encoding a cytosolic exporter, it is reasonable to assume that both products are secreted by the same protein. 


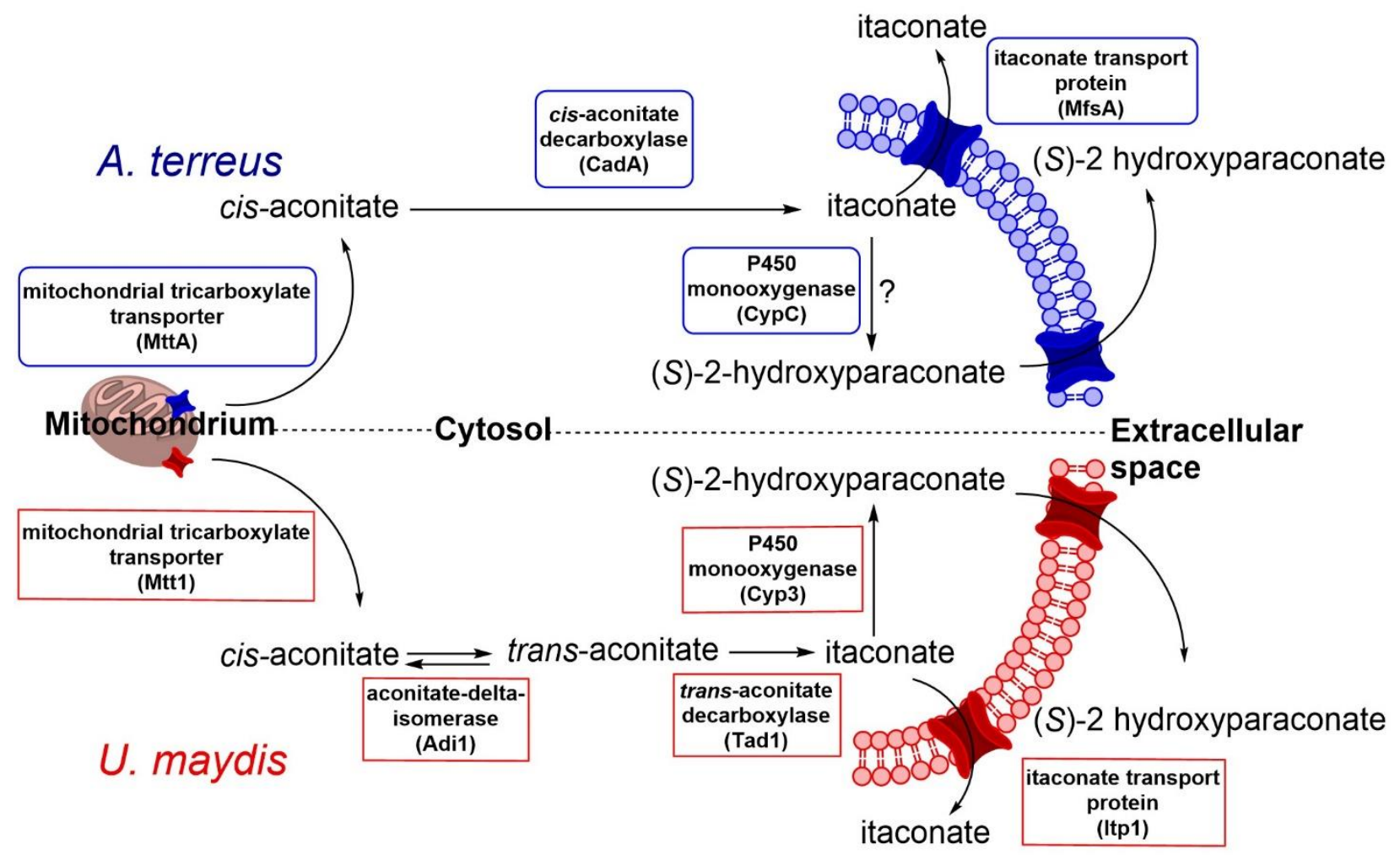

Fig. 1. Comparison of the itaconate and (S)-2-hydroxyparaconate biosynthesis pathways of $U$. maydis and A. terreus.

Although the functions of the transporters involved in the production of itaconate and its derivative (S)-2-hydroxyparaconate are likely similar in both U. maydis and A. terreus, the differences in their protein sequence, and their underlying biosynthetic pathways, may affect their specific activity and/or substrate affinity. In addition, the mechanism of (S)-2-hydroxyparaconate transport has thus far not been investigated. Thus, the aim of this study is to characterize these transporter proteins in the unicellular fungus $U$. maydis and to investigate their influence on the production of itaconate and $(S)$-2-hydroxyparaconate.

\section{Experimental procedures}

\subsection{Strains and culture conditions}

All strains used in this work are listed in

Table. 2. To constitutively express the transporter genes $m t t A$ (ATEG_09970) and $m f s A$ (ATEG_09972) in Ustilago maydis, these genes were synthesized in vitro (GeneArt Life Technologies, Regensburg, Germany). Since U. maydis tends to pre-maturely polyadenlylate heterologous mRNA (Zarnack et al., 2006), the sequence was di-codon optimized via the dicodon optimizer (http://dicodonoptimization.appspot.com/). Repeats of $\geq 8$ bp length were manually edited. The full synthetic sequences are shown in the supplemental data D1 and D2. The synthesized genes were subcloned into vector 
pETEF_GFP_CBX_Q018 (Sarkari et al., 2014; Spellig et al., 1996) using BamHI and NotI restriction sites resulting in the plasmids pETEF_CbxR_At_mttA and pETEF_CbxR_At_mfsA (Fig. S1). The constructs were confirmed by sequencing by Life Technologies, Germany. Plasmids were transformed via protoplast transformation according to Schulz et al. (1990) and transformants were selected for resistance against carboxin. Integration of $m t t A$ and $m f s A$ was verified by PCR and single integration was confirmed via southern blotting resulting in the strains listed in

Table. 2.

Shaking cultures of $U$. maydis mutants were performed in System Duetz ${ }^{\circledR}$ (24 Well plates) with a filling volume of $1.5 \mathrm{~mL}\left(\mathrm{~d}=50 \mathrm{~mm}, \mathrm{n}=300 \mathrm{rpm}, \mathrm{T}=30^{\circ} \mathrm{C}\right.$ and $\Phi=80 \%$ ) (Duetz et al., 2000). The screening medium contained $50 \mathrm{~g} \mathrm{~L}^{-1}$ glucose, $0.8 \mathrm{~g} \mathrm{~L}^{-1} \mathrm{NH}_{4} \mathrm{Cl}, 0.2 \mathrm{~g} \mathrm{~L}^{-1} \mathrm{MgSO}_{4} \cdot 7 \mathrm{H}_{2} \mathrm{O}, 0.01 \mathrm{~g} \mathrm{~L}^{-1} \mathrm{FeSO}_{4} \cdot 7 \mathrm{H}_{2} \mathrm{O}, 0.5$ $\mathrm{g} \mathrm{L}^{-1} \mathrm{KH}_{2} \mathrm{PO}_{4}, 1 \mathrm{~mL} \mathrm{~L}{ }^{-1}$ vitamin solution, $1 \mathrm{~mL} \mathrm{~L}^{-1}$ trace element solution, and as buffer various concentrations of 2-(N-morpholino) ethanesulfonic acid (MES) and $33 \mathrm{~g} \mathrm{~L}^{-1} \mathrm{CaCO}_{3}$ (Geiser et al., 2016). Cultures were parallel inoculated into multiple microtiter plates (System Duetz ${ }^{\circledR} 24$ well plates) and for each sample point a complete plate was taken as sacrificial sample in order to ensure continuous oxygenation. Controlled batch cultivations were performed in a New Brunswick BioFlo® 110 bioreactor (Eppendorf, Germany) with a total volume of $1.3 \mathrm{~L}$ and a working volume of $0.5 \mathrm{~L}$. All cultivations were performed in batch medium containing $200 \mathrm{~g} \mathrm{~L}^{-1}$ glucose, $4 \mathrm{~g} \mathrm{~L}^{-1} \mathrm{NH}_{4} \mathrm{Cl}, 0.2 \mathrm{~g} \mathrm{~L}^{-1} \mathrm{MgSO}_{4} \cdot 7 \mathrm{H}_{2} \mathrm{O}, 0.01 \mathrm{~g} \mathrm{~L}^{-1} \mathrm{FeSO}_{4} \cdot 7 \mathrm{H}_{2} \mathrm{O}$, $0.5 \mathrm{~g} \mathrm{~L}^{-1} \mathrm{KH}_{2} \mathrm{PO}_{4}, 1 \mathrm{~g} \mathrm{~L}^{-1}$ yeast extract (Merck Millipore, Germany), $1 \mathrm{~mL} \mathrm{~L}^{-1}$ vitamin solution, and $1 \mathrm{ml} \mathrm{L}^{-}$ ${ }^{1}$ trace element solution. During cultivation, $\mathrm{pH} 6.0$ was maintained by automatic addition of $10 \mathrm{M} \mathrm{NaOH}$ and the dissolved oxygen tension (DOT) was kept constant at approximately $80 \%$ saturation by automatic adjustment of the stirring rate (700-1200 rpm). The bioreactor was aerated with an aeration rate of $1 \mathrm{~L} \mathrm{~min}^{-}$ ${ }^{1}$ ( $\left.2 \mathrm{vvm}\right)$, while evaporation was limited by sparging the air through a water bottle. The temperature was set at $30^{\circ} \mathrm{C}$. The bioreactor was inoculated to a final $\mathrm{OD}_{600}$ of 0.75 with washed cells from an overnight culture in $50 \mathrm{~mL}$ screening medium.

\subsection{Analytical methods}

Cell densities were measured by determining the absorption at $600 \mathrm{~nm}$ with an Ultrospec 10 Cell Density Meter (Amersham Biosciences, Chalfont St Giles, UK).

The ammonium concentration in the culture supernatant was measured by a colorimetric method according to (Willis et al., 1996) using salicylate and nitroprusside.

Itaconate, $(S)$-2-hydroxyparaconate, and glucose in the supernatants were analyzed in a Beckmann Coulter System Gold High Performance Liquid Chromatography (Beckmann Coulter GmbH, Germany) with an Organic Acid Resin $300 \times 8 \mathrm{~mm}$ column (CS-Chromatography, Germany), a differential refractometer LCD 201 (MELZ, Germany) and UV/VIS detector (Thermo Fischer, Germany). As eluent $5 \mathrm{mM} \mathrm{H}_{2} \mathrm{SO}_{4}$ with a flow rate of $0.6 \mathrm{~mL} \mathrm{~min}^{-1}$ and a temperature of $40^{\circ} \mathrm{C}$ was used. All samples were filtered with Rotilabo® syringe filters (CA, $0.20 \mu \mathrm{m}, \varnothing 15 \mathrm{~mm}$ ) and afterwards diluted 1:5 with $5 \mathrm{mM} \mathrm{H}_{2} \mathrm{SO}_{4}$. All components were identified via retention time and UV/RI quotient compared to corresponding standards. All values are the arithmetic mean of at least two biological replicates. Error bars indicate the deviation from the mean for $\mathrm{n}$ $=2$, if $\mathrm{n}>2$ error bars indicate the standard error of the mean. 
For (S)-2-hydroxyparaconate standards, samples of previous studies were used, where (S)-2hydroxyparaconate was synthesized and purified (Geiser et al., 2016). Since the purity ( 70\%) of these samples is not exactly known, indicated $(S)$-2-hydroxyparaconate values should be taken as rough estimates only.

Table. 2. U. maydis strains used in this study

\begin{tabular}{|c|c|c|}
\hline Strain designation & Resistance & Reference \\
\hline $\begin{array}{l}\text { Ustilago maydis DSM17144 } \\
\text { (Ustilago maydis MB215) }\end{array}$ & & (Hewald et al., 2005) \\
\hline Ustilago maydis $\mathrm{MB} 215 \Delta U m \_m t t 1$ & Hygromycin & (Geiser et al., 2015) \\
\hline Ustilago maydis $\mathrm{MB} 215 \Delta U m \_m t t 1+\mathrm{P}_{\text {etef }} U m \_m t t 1$ & $\begin{array}{l}\text { Hygromycin } \\
\text { Carboxin }\end{array}$ & (Przybilla, 2014) \\
\hline Ustilago maydis $\mathrm{MB} 215+\mathrm{P}_{\text {etef }} U m \_m t t 1$ & Carboxin & this study \\
\hline Ustilago maydis $\mathrm{MB} 215 \Delta U m \_m t t 1+\mathrm{P}_{\text {etef }} A t \_m t t A$ & $\begin{array}{l}\text { Hygromycin } \\
\text { Carboxin }\end{array}$ & this study \\
\hline Ustilago maydis $\mathrm{MB} 215+\mathrm{P}_{\text {etef }} A t \_m t t A$ & Carboxin & this study \\
\hline Ustilago maydis MB215 $\Delta U$ Um_itp1 $_{1}$ & Hygromycin & (Geiser et al., 2015) \\
\hline Ustilago maydis MB215 $\Delta U m_{-}$itpl+ $\mathrm{P}_{\text {etef }} U m_{-}$itpl & $\begin{array}{l}\text { Hygromycin } \\
\text { Carboxin }\end{array}$ & (Przybilla, 2014) \\
\hline Ustilago maydis $\mathrm{MB} 215+\mathrm{P}_{\text {etef }} U m \_$itpl & Carboxin & (Geiser et al., 2015) \\
\hline Ustilago maydis $\mathrm{MB} 215 \Delta U m_{-}$itpl+P $\mathrm{P}_{\text {etef }} A t \_m f s A$ & $\begin{array}{l}\text { Hygromycin } \\
\text { Carboxin }\end{array}$ & this study \\
\hline Ustilago maydis $\mathrm{MB} 215+\mathrm{P}_{\text {etef }} A t \_m f s A$ & Carboxin & this study \\
\hline
\end{tabular}

\section{Results and discussion}

\subsection{Comparing the role of Itp1 from $U$. maydis and MfsA from A. terreus in itaconic acid production}

Both Ustilago maydis and Aspergillus terreus can oxidize itaconate to (S)-2-hydroxyparaconate, but they each only carry one gene encoding an Mfs-type cytoplasma membrane transporter in their itaconate gene clusters. In order to compare the exporters of these two genera in the same biological background, both At_mfsA from A. terreus and Um_itpl from U. maydis were expressed under control of the $\mathrm{P}_{\text {etef }}$ promoter in U. maydis MB215 $\Delta U m_{-} i t p 1$. The At_mfsA gene was dicodon-optimized to enable expression in $U$. maydis (Zarnack et al., 2006). To ensure comparability, the constructs were integrated into the ip-locus and single copy insertions were selected by diagnostic PCR and Southern blot analysis. Subsequently, in targeted single copy transformants, itaconate and (S)-2-hydroxyparaconate production, glucose consumption, and $\mathrm{OD}_{600}$ were analyzed in order to elucidate the role of the exporters encoded by At_mfsA from A. terreus and 
Um_itpl from U. maydis in the corresponding U. maydis deletion strain (MB215 $\left.\Delta U m \_i t p 1\right)$ (Fig. 2A, D, C, F). Surprisingly, although the expression of At_mfsA did lead to a slight itaconate production, it did not fully restore the production of $U$. maydis $\Delta U m_{-} i t p l$ to the level of the wildtype (Fig. 2A). In contrast, the control strain $U$. maydis $\Delta U m_{-} i t p 1+\mathrm{P}_{\text {etef }} U m_{-}$itpl produced 1.4-fold more itaconate than the wildtype (Fig. 2A). Conversely, $\Delta U m_{-}$itp1+P $\mathrm{P}_{\text {etef }} U m_{-}$itpl produced less (S)-2-hydroxyparaconate than the wildtype, while $\Delta U m_{-} i t p 1+\mathrm{P}_{\text {etef }} A t \_m f s A$ produced 1.7-fold more $(S)$-2-hydroxyparaconate than the wildtype (Fig. $\left.2 \mathrm{D}\right)$. The $\mathrm{P} 450$ monooxygenase encoded by cyp3 converts itaconate to (S)-2-hydroxyparaconate in the cytoplasm (Geiser et al., 2016). The fact that complementation with both transporters significantly elevate the extracellular concentration of both products in comparison to the $\Delta U m_{-} i t p l$ deletion strain indicates that $U m \_I t p 1$ and $A t \_$MfsA can both transport itaconate as well as $(S)$-2-hydroxyparaconate. However, when both products are produced simultaneously, our data suggests that competitive inhibition between these metabolites takes place, with $U m \_I t p 1$ having a higher affinity for itaconate, and At_MfsA favoring $(S)$-2hydroxyparaconate (Table. 1) (Fig. 2B, E).

An explanation for the low concentration of (S)-2-hydroxyparaconate produced by $\Delta U m_{-}$itp $1+\mathrm{P}_{\text {etef }} U m_{-}$itpl compared to the wildtype could be that the overexpression of Um_Itp1 with the strong and constitutive promoter $\mathrm{P}_{\text {etef }}$ enables a more efficient secretion of itaconate, thereby lowering its concentration in the cytoplasm. This would lower the substrate concentration of Cyp3, reducing the $(S)$-2-hydroxyparaconate production rate as long as the enzyme is not operating under substrate-saturating conditions. The reverse effect likely occurs in $U$. maydis $\Delta U m_{-} i t p 1+\mathrm{P}_{\text {etef }} A t \_m f s A$, resulting in higher itaconate and lower $(S)$-2-hydroxyparaconate concentrations in the cytoplasm, thereby increasing Cyp3 activity. In this way, product export and the metabolic rates of the branched production pathway are inherently linked. Although this is likely an important driver for product specificity in the natural habitats of U. maydis and A. terreus, it may be less relevant in optimized itaconate production strains, which don't produce (S)-2-hydroxyparaconate. However, it does suggest that the $\mathrm{K}_{\mathrm{m}}$ value of At_MfsA for itaconate is higher than that of $U m \_$Itp1. If this is indeed the case, the resulting elevated cytosolic itaconate concentration could lead to product inhibition of CadA or Tad1, suggesting that $U m$ Itp1 could be a better itaconate exporter even if $(S)$-2-hydroxyparaconate is not produced. 

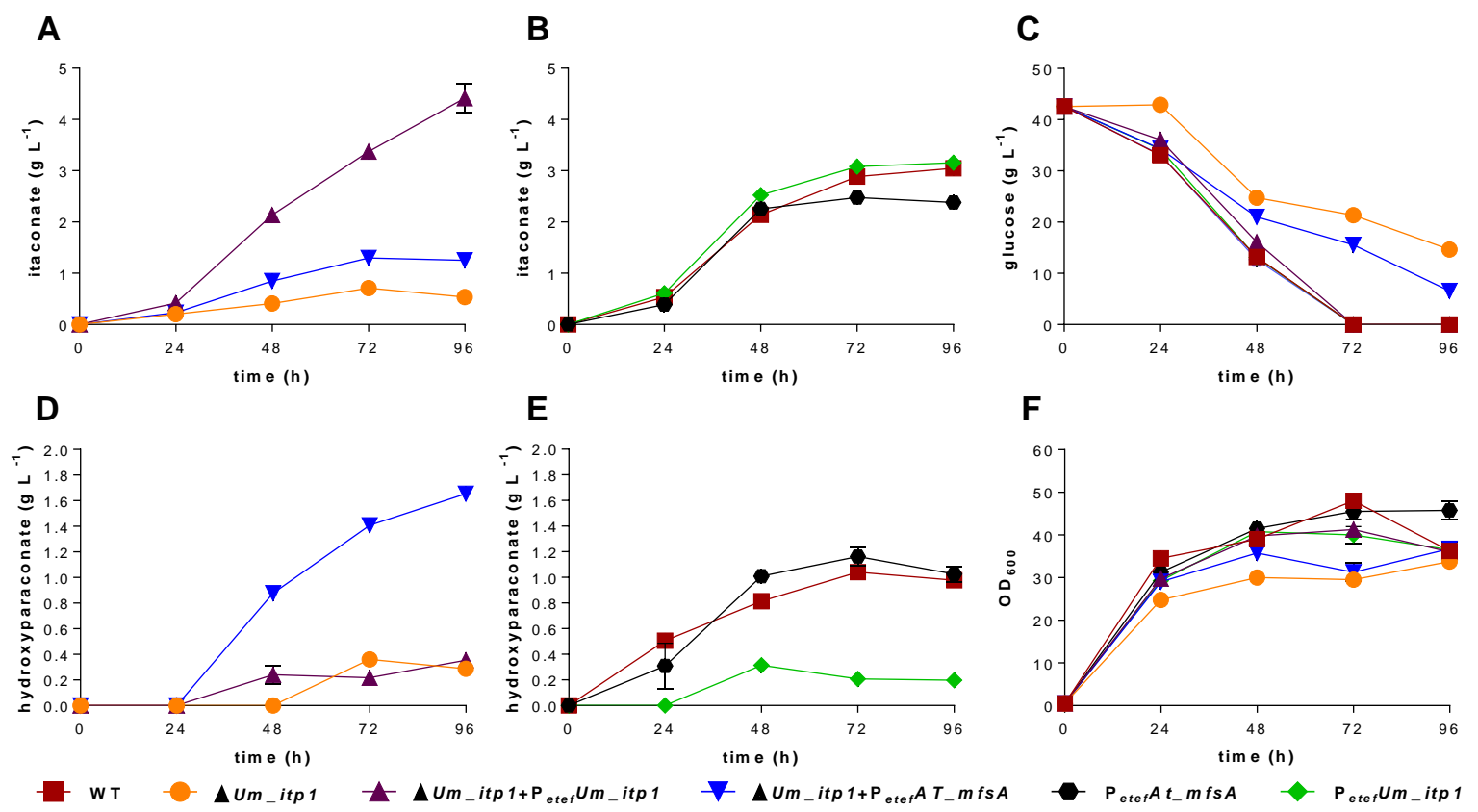

Fig. 2. Acid production and growth of various U. maydis strains expressing itaconate transport proteins. Itaconate (A-B), (S)-2-hydroxyparaconate (D-E), and glucose concentration (C), as well as growth $\left(\mathrm{OD}_{600}\right)$ (F) of itpl / $m f s A$ expressing mutants in comparison to wildtype and $\Delta U m_{-} i t p 1$ controls during System Duetz $^{\circledR}$ cultivation in screening medium. Error bars indicate the standard error of the mean $(n=4)$.

\subsection{Comparing the role of Mtt1 from $U$. maydis and MttA from A. terreus in itaconic acid production}

In order to quantitatively compare mitochondrial transporters in the same biological background, both At_mttA from A. terreus and $U m \_m t t 1$ from $U$. maydis were expressed under control of the $\mathrm{P}_{\text {etef }}$ promoter in $U$. maydis MB215 $\Delta U m \_m t t 1$ in the corresponding $U$. maydis deletion strain (MB215 $\left.\Delta U m \_m t t A\right)$ as described above for $m f s A$. Both constructs complemented the itaconate producing phenotype of the $\Delta U m \_m t t 1$ deletion strain, confirming that $A t \_M t t A$ and $U m \_M t t 1$ have a similar biochemical function. The complementation strains reached higher maximum titers than the wildtype (Fig. 3 A, D), likely due to the higher expression from the strong, constitutive $\mathrm{P}_{\text {etef }}$ promoter (Geiser et al., 2016; Sarkari et al., 2014; Zambanini et al., 2017b). However, U. maydis $\Delta U m \_m t t 1+\mathrm{P}_{\text {etef }} A t \_m t t A$ produced 1.3 -fold more itaconate than $U$. maydis $\Delta U m \_m t t 1+\mathrm{P}_{\text {etef }} U m \_m t t 1$ (Fig. 3A). The production rates of $(S)$-2-hydroxyparaconate in the complementation strains were also increased, although the maximum titer was not affected (Tab.1). The fact that the strain expressing At_mttA produces more itaconate indicates that the mitochondrial transporter from A. terreus can sustain a higher cis-aconitate flux compared to Mtt1 from U. maydis. An alternative explanation would be that the two mitochondrial carriers have different antiport substrates. Circumstantial evidence suggests that cis-aconitate is mostly likely exchanged for malate by At_MttA (Jaklitsch et al., 1991; Steiger et al., 2016). The fact that this protein can complement a $\Delta U m \_m t t 1$ deletion suggests that the $U$. maydis protein transports the same substrates, while the improved itaconate production with At_MttA suggests that this protein either has a higher $\mathrm{V}_{\max }$ or a lower $\mathrm{K}_{\mathrm{m}}$. 

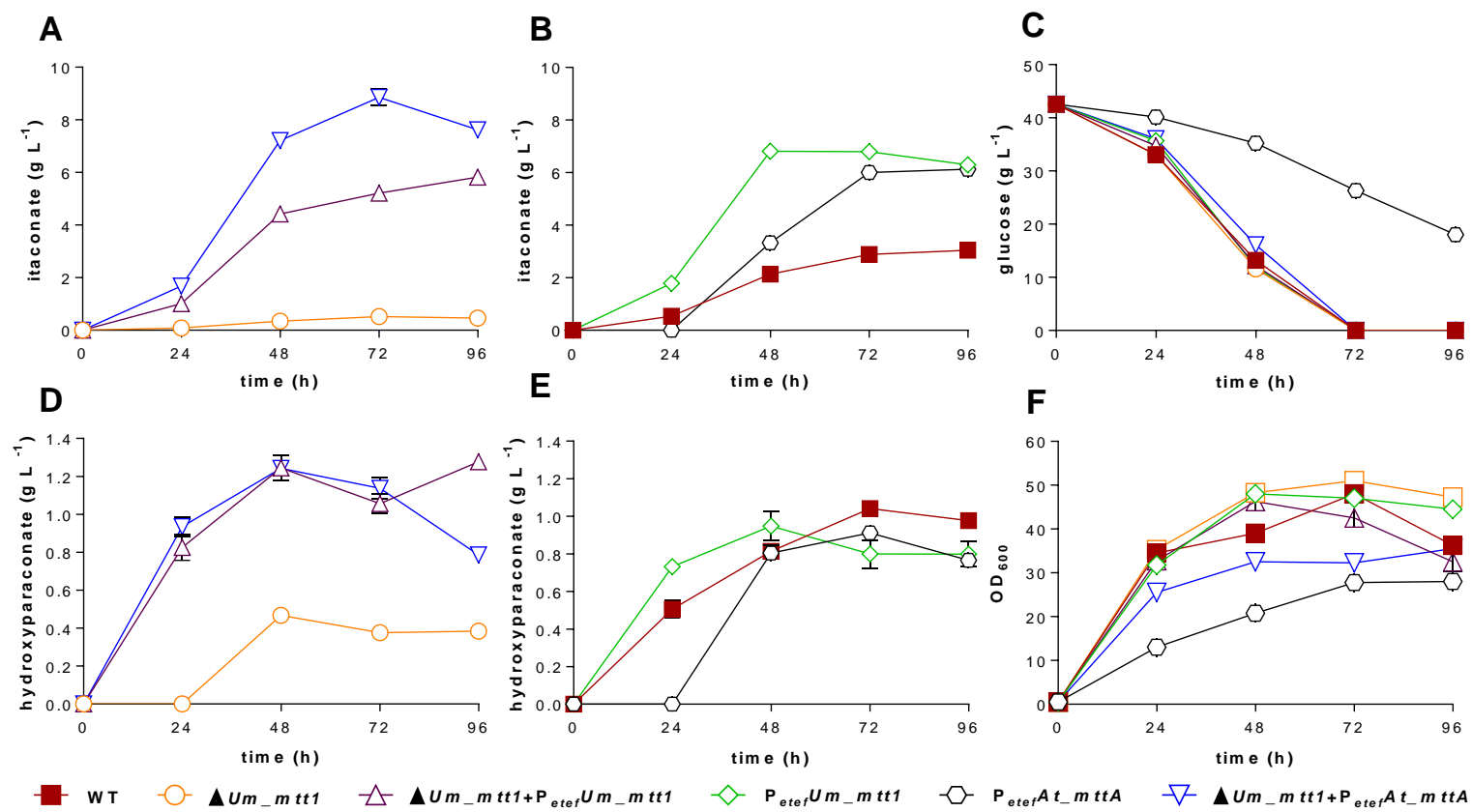

Fig. 3. Acid production and growth of various $U$. maydis strains expressing mitochondrial transporters. Itaconate (A-B), (S)-2-hydroxyparaconate (D-E), and glucose concentration (C), as well as growth $\left(\mathrm{OD}_{600}\right)$ (F) of $m t t 1 / m t t A$ expressing mutants in comparison to wildtype and $\Delta U m \_m t t 1$ controls during System Duetz $^{\circledR}$ cultivation in screening medium is shown. Error bars indicate thr standard error of the mean $(n=4)$.

Given the already higher titers of these complementation strains, we sought to further improve itaconate and/or (S)-2-hydroxyparaconate production by selecting transformants with multicopy insertions of $\mathrm{P}_{\text {etef } A t \_m t t A}$ in wildtype $U$. maydis MB215. However, this resulted in a decreased production of itaconate compared to the complementation strain $\Delta U m \_m t t 1+\mathrm{P}_{\text {etef }} \mathrm{At} \_m t t A$ (Fig 3. B). Biomass growth and glucose consumption were strongly decreased in the overexpression strain (Fig. 3C, F). Closer inspection of the results obtained with the $\Delta U m \_m t t 1+A t \_m t t A$ complementation strain also showed growth retardation, although less drastic (Fig. 3F). Similar growth defects were observed by Huang et al. (2014) upon overexpression of $m t t A$ in A. terreus, even though itaconate production was not increased in this strain. A burden of membrane protein overexpression per se is unlikely since a control strain with multiple copies of $\mathrm{P}_{\text {etef }} U m \_m t t 1$ in wildtype $U$. maydis MB215 didn't show this growth defect. In wildtype $U$. maydis, genes of the itaconate cluster are induced upon the depletion of nitrogen from the medium (Geiser et al., 2015; Maassen et al., 2014; Zambanini et al., 2017a), thereby separating itaconate production from biomass growth. The use of the constitutive $\mathrm{P}_{\text {etef }}$ promoter for expression of At_mttA leads to the production of MttA in the growth phase, thus posing a drain of the central metabolite cis-aconitate from the mitochondria, which is likely responsible for the growth defect. The fact that this was not observed upon overexpression of $U m \_M t t 1$ is in line with the other indications that $A t \_M t t A$ is a more effective transporter of cis-aconitate. To evaluate the potential of $U$. maydis $\Delta U m \_m t t 1+\mathrm{P}_{\text {etef }} A t \_m t t A$ for itaconate production, this strain was cultured in $\mathrm{pH}$ controlled high-density pulsed fed-batch fermentations with $4 \mathrm{~g} \mathrm{~L}^{-1} \mathrm{NH}_{4} \mathrm{Cl}$ and a starting concentration of $188 \pm 1.3 \mathrm{~g} \mathrm{~L}^{-1}$ glucose (Fig. 4). Under these conditions the engineered strain produced 33 $\pm 3 \mathrm{~g} \mathrm{~L}^{-1}$ itaconate and an estimated $24 \pm 2 \mathrm{~g} \mathrm{~L}^{-1}(S)$-2-hydroxyparaconate after $190 \mathrm{~h}$. Under similar conditions wildtype $U$. maydis MB215 produces $14.0 \pm 0.3 \mathrm{~g} \mathrm{~L}^{-1}$ itaconate and $21.3 \pm 0.7 \mathrm{~g} \mathrm{~L}^{-1}(S)-2$ hydroxyparaconate with similar ammonium consumption and growth (Geiser et al., 2016). U. maydis 
$\Delta U m \_m t t 1+\mathrm{P}_{\text {etef }} A t \_m t t A$ produced itaconate at an overall rate of $0.29 \pm 0.00 \mathrm{~g} \mathrm{~L}^{-1} \mathrm{~h}^{-1}$ and a yield of $0.15 \pm$ $0.00 \mathrm{~g}_{\text {ita }} \mathrm{gglc}^{-1}$, which is 2.1 -fold, respectively 3.6-fold higher than the wildtype. It is interesting to note that both in shaken cultures and in controlled fed-batch, the engineered strains didn't produce more $(S)$-2hydroxyparaconate, even when itaconate production was increased. This indicates that the Cyp3 P450 monooxygenase that catalyzes the conversion of itaconate to $(S)$-2-hydroxyparaconate cannot support a higher flux. In total, $U$. maydis $\Delta U m \_m t t 1+$ PetefAt_mttA produced $0.29 \mathrm{~g} \mathrm{~L}^{-1} \mathrm{~h}^{-1}$ acids (itaconate $+(S)-2$ hydroxyparaconate), which is comparable to itaconate production values achieved by Geiser et al. (2016) after overexpression of rial and deletion of cyp3. The combination of these modifications with the tuned overexpression of $A t$ t $m t t A$ could further enhance itaconate production without $(S)$-2-hydroxyparaconate as a by-product.

Table. 1. Production parameters of $U$. maydis MB215 and transporter mutants during System Duetz ${ }^{\circledR}$ cultivation in screening medium is shown. Errors indicate the standard error of the mean $(n=4)$.

\begin{tabular}{|c|c|c|c|c|c|c|}
\hline & & & & $/ s^{\mathrm{b}}$ & & $\begin{array}{l}r_{p} \\
\left.-1 h^{-1}\right)^{c}\end{array}$ \\
\hline & ITA $^{d}$ & $2-\mathrm{HP}^{\mathrm{e}}$ & ITA & 2-HP & ITA & 2-HP \\
\hline WT & $3.0 \pm 0.1$ & $1.0 \pm 0.0$ & $0.07 \pm 0.00$ & $0.02 \pm 0.00$ & $0.03 \pm 0.00$ & $0.003 \pm 0.004$ \\
\hline$\Delta U m_{-} i t p 1$ & $0.7 \pm 0.1$ & $0.4 \pm 0.1$ & $0.02 \pm 0.00$ & $0.01 \pm 0.00$ & $0.01 \pm 0.00$ & $0.003 \pm 0.001$ \\
\hline$\Delta U m_{-} i t p 1+P_{\text {etef }} U m_{-} i t p 1$ & $4.4 \pm 0.5$ & $0.4 \pm 0.1$ & $0.10 \pm 0.01$ & $0.01 \pm 0.00$ & $0.05 \pm 0.00$ & $0.004 \pm 0.001$ \\
\hline PetefUm_itp1 & $3.2 \pm 0.2$ & $0.2 \pm 0.0$ & $0.07 \pm 0.00$ & $0.00 \pm 0.00$ & $0.03 \pm 0.00$ & $0.002 \pm 0.000$ \\
\hline$\Delta U m \_i t p 1+P_{\text {etef }} A T \_m f s A$ & $1.3 \pm 0.1$ & $1.7 \pm 0.1$ & $0.03 \pm 0.00$ & $0.05 \pm 0.00$ & $0.01 \pm 0.00$ & $0.02 \pm 0.00$ \\
\hline Petef $A T \_m f s A$ & $2.5 \pm 0.1$ & $1.2 \pm 0.1$ & $0.06 \pm 0.00$ & $0.02 \pm 0.00$ & $0.02 \pm 0.00$ & $0.01 \pm 0.00$ \\
\hline$\Delta U m_{-} m t t 1$ & $0.5 \pm 0.0$ & $0.5 \pm 0.0$ & $0.01 \pm 0.00$ & $0.01 \pm 0.00$ & $0.00 \pm 0.00$ & $0.00 \pm 0.00$ \\
\hline$\Delta U m_{-} m t t 1+P_{\text {etef }} U m_{-} m t t 1$ & $5.8 \pm 0.2$ & $1.3 \pm 0.0$ & $0.14 \pm 0.00$ & $0.03 \pm 0.00$ & $0.06 \pm 0.00$ & $0.01 \pm 0.00$ \\
\hline PetefUm_mtt1 & $6.8 \pm 0.0$ & $0.9 \pm 0.1$ & $0.15 \pm 0.01$ & $0.02 \pm 0.00$ & $0.07 \pm 0.00$ & $0.01 \pm 0.00$ \\
\hline PetefAT_mttA & $6.1 \pm 0.2$ & $0.9 \pm 0.1$ & $0.25 \pm 0.01$ & $0.03 \pm 0.00$ & $0.06 \pm 0.00$ & $0.01 \pm 0.00$ \\
\hline$\Delta U m \_m t t 1+P_{\text {etef }} A T \_m t t A$ & $8.8 \pm 0.6$ & $1.2 \pm 0.0$ & $0.18 \pm 0.00$ & $0.02 \pm 0.00$ & $0.08 \pm 0.00$ & $0.01 \pm 0.00$ \\
\hline
\end{tabular}

${ }^{a}$ titer $_{\text {max }}$; maximum titer.

${ }^{\mathrm{b}} \mathrm{Y}_{\mathrm{P} / \mathrm{S}}$; overall yield product per consumed glucose.

${ }^{\mathrm{c}} \mathrm{r}_{\mathrm{p}}$; overall production rate.

d ITA; itaconate

e 2-HP; (S)-2-hydroxyparaconate 

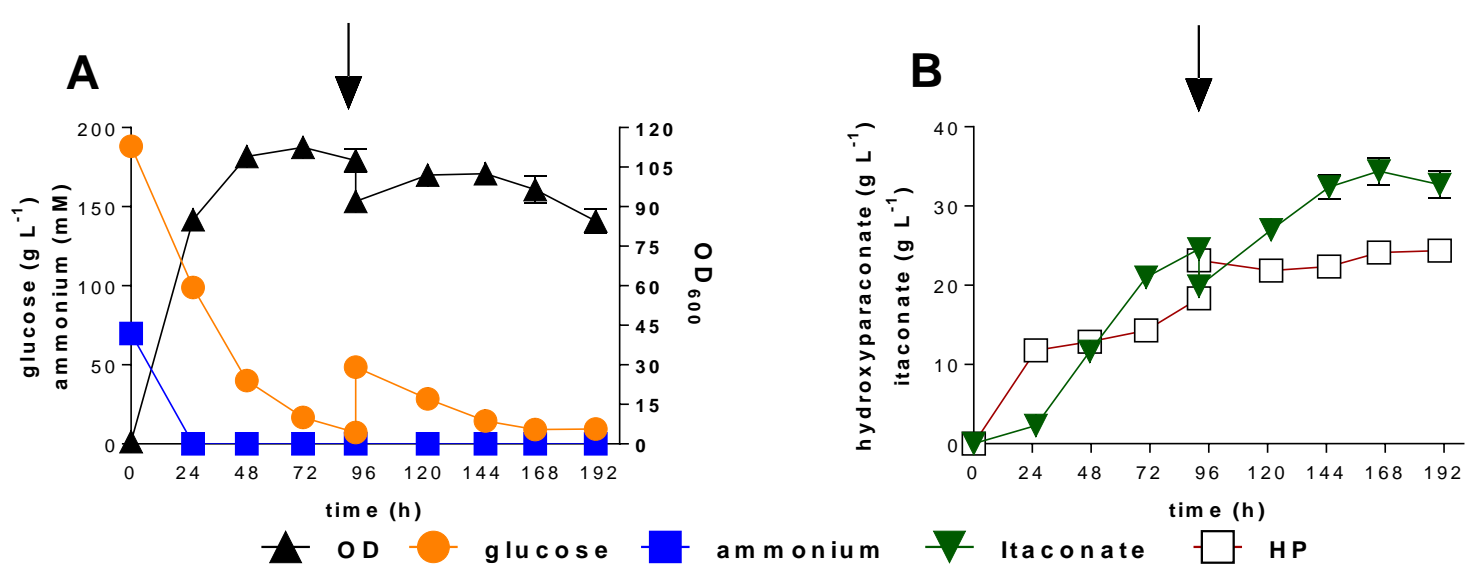

Fig. 4. Controlled high-density pulsed fed-batch fermentation of $U$. maydis $\Delta U m \_m t t 1+\mathrm{P}_{\text {etef }} A T \_m t t A$. A: $\mathrm{OD}_{600}(\boldsymbol{\Delta})$, glucose $(\bullet)$ and ammonium concentration $(\boldsymbol{\bullet})$ and B: Concentration of itaconate $(\boldsymbol{\nabla})$ and $(S)-2-$ hydroxyparaconate $(\square)$ during fermentation in a bioreactor containing batch medium with $200 \mathrm{~g} \mathrm{~L}^{-1}$ glucose, $4 \mathrm{~g} \mathrm{~L}^{-1} \mathrm{NH}_{4} \mathrm{Cl}$ at $\mathrm{pH} 6.0$ titrated with $\mathrm{NaOH}$. Arrows indicate addition of $100 \mathrm{~mL}$ of $50 \%$ glucose. Error bars indicate the standard error of the mean $(n=4)$.

\subsection{Transporters involved in itaconate production do not affect $\mathrm{pH}$ optimum}

The optimal extracellular $\mathrm{pH}$ for itaconate production of A. terreus and $U$. maydis is very different. With A. terreus, itaconic acid is usually produced at a $\mathrm{pH}$ below 3.8, and the optimal $\mathrm{pH}$ range is mostly governed by limitations in cell morphology and pathway induction (Karaffa et al., 2015; Krull et al., 2017; Kuenz et al., 2012). In contrast, wildtype $U$. maydis only produces itaconate at a $\mathrm{pH}$ above 5.5 (Geiser et al., 2014). One hypothesis could be that transporters involved in itaconate production, as key interaction points between cellular compartments and the intracellular and extracellular space, might be responsible for this difference. To test this hypothesis, the engineered complementation strains were cultivated in screening media with different buffers. The use of $33 \mathrm{~g} \mathrm{~L}^{-1} \mathrm{CaCO}_{3}$ maintains $\mathrm{pH}$ values above 6 (Fig. 5D). In contrast, by varying the concentration of a soluble MES buffer between 20 and $100 \mathrm{mM}$, the $\mathrm{pH}$ decreases to different extents during growth and itaconate production (Fig $5 \mathrm{~A}, \mathrm{~B}, \mathrm{C}$ ). In wildtype U. maydis MB215, this results in the production of different final concentrations of itaconate, due to the difference in the concentration where $\mathrm{pH}$ drops below 5.5 and inhibits further production (Geiser et al., 2014). If the expression of the A. terreus transporters affects the $\mathrm{pH}$ optimum for itaconate production by $U$. maydis, the relative itaconate concentrations of theses strains are expected to be higher than those of the controls expressing the native $U$. maydis transporters in the media with low buffer concentrations. However, this effect is not observed. Instead, the same trend of lower itaconate production with lower buffer capacity is observed for all strains (Fig. $5 \mathrm{~B}, \mathrm{E}$ ). However, strains which produce more itaconate in $\mathrm{CaCO}_{3}$ buffer, such as U. maydis $\Delta U m \_m t t 1+\mathrm{P}_{\text {etef }} \mathrm{At} \_m t t A$, also consistently make more acids with limiting buffer concentrations $(40,70$, and $100 \mathrm{mM}$ MES). Such overproducing strains also reach a lower final $\mathrm{pH}$, indicating that a low $\mathrm{pH}$ per se does not inhibit itaconate and (S)-2-hydroxyparaconate production in U. maydis (Fig. 5B, C, E, F). One explanation for this could be that the inhibition of itaconate production at low $\mathrm{pH}$ values is the result of a dynamic equilibrium between uptake and secretion. Below pH 5.5, a significant fraction of fully protonated itaconic acid $\left(\mathrm{pKa}_{1}=3.8\right)$ is formed. This protonated form, and not the dissociated from, can freely diffuse across the cytoplasmic membrane, facilitating itaconate reuptake into the cell (Geiser et al., 2016). Lower $\mathrm{pH}$ values will lead to higher extracellular concentrations of the protonated form, thus increasing the 
diffusion-driven reuptake rate. This will reduce the net production of itaconate to zero when the reuptake rate equals the production rate, leading to an accumulation of intracellular itaconate, which would likely inhibit its own production, and also places a burden on the maintenance of cytoplasmic $\mathrm{pH}$ homeostasis through weak acid uncoupling. This mechanism would explain why strains with a higher itaconate production rate can sustain a lower extracellular $\mathrm{pH}$. In addition, the increase in intracellular itaconate would also enhance the rate of intracellular oxidation to $(S)$-2-hydroxyparaconate, while the reuptake of this oxidation product is lower than that of itaconate due to its lower $\mathrm{pK}_{\mathrm{a}}$ value of 2.8 (Guevarra \& Tabuchi, 1990a).
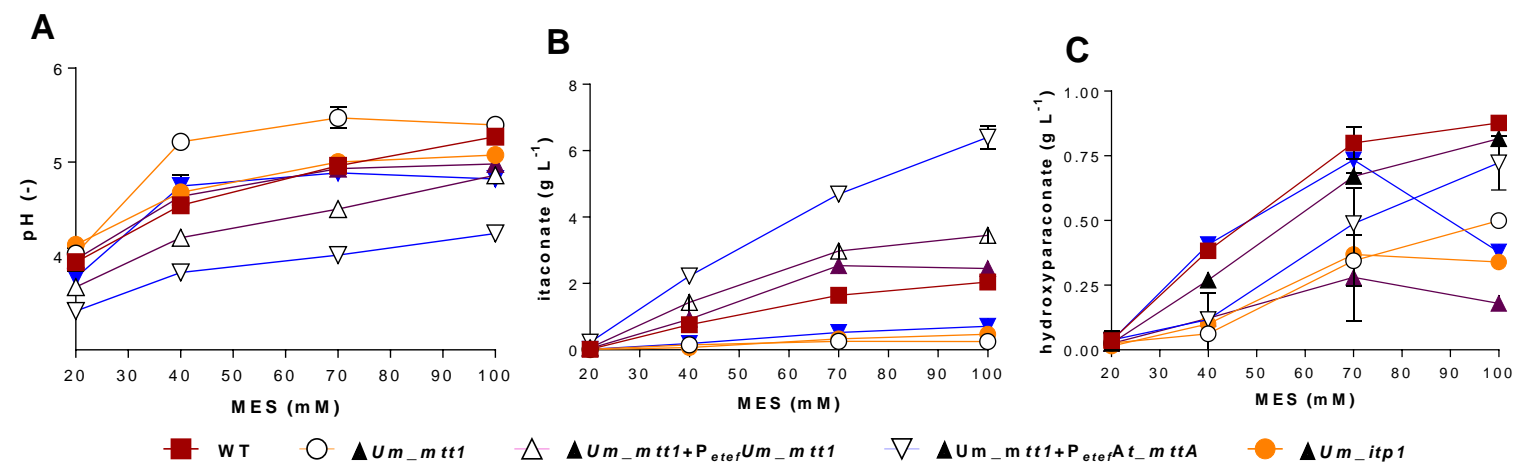

$-\Delta U m_{-} i t p 1+\mathrm{P}_{\text {etef }} U m_{-} i t p 1 \rightarrow \Delta U m_{-} i t p 1+\mathrm{P}_{\text {ete }} A t t_{-} m f s A$
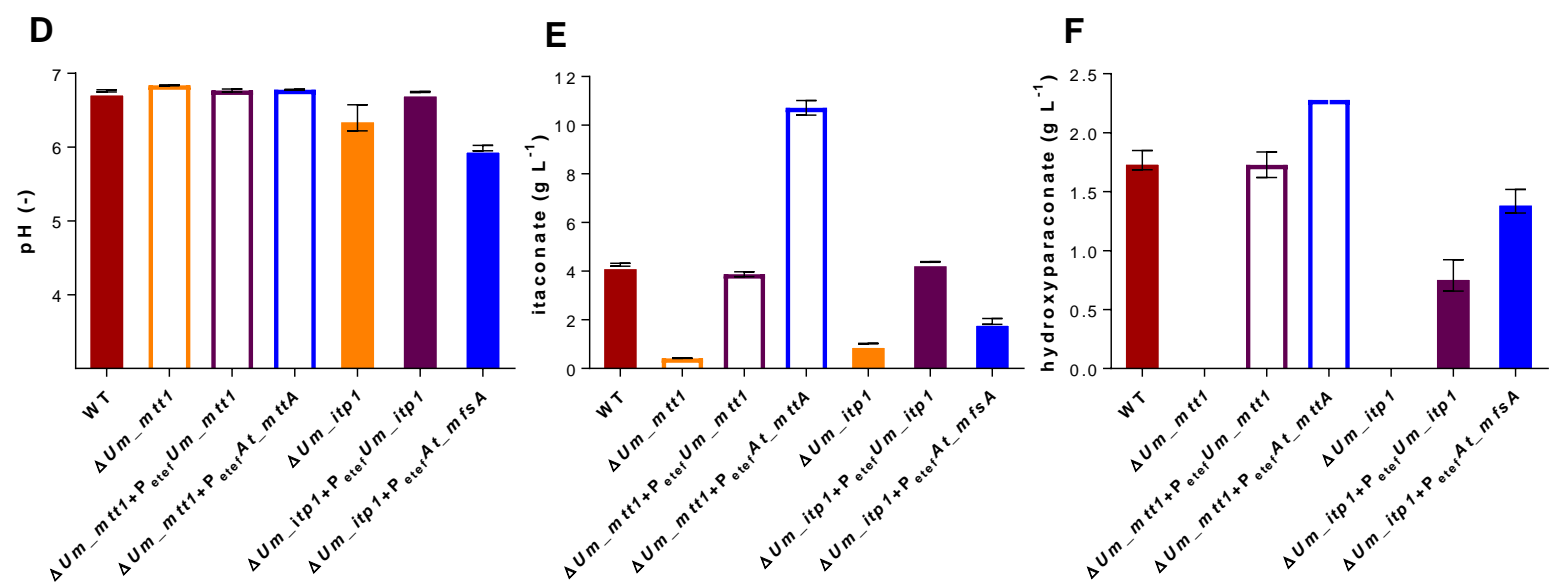

Fig. 5. Influence of buffer concentration on itaconate and (S)-2-hydroxyparaconate production by various $U$. maydis MB215 mutants. $\mathrm{pH}$ values $(\mathrm{A}, \mathrm{D})$, itaconate $(\mathrm{B}, \mathrm{E})$, and $(S)$-2-hydroxyparaconate $(\mathrm{C}, \mathrm{F})$ concentrations after $72 \mathrm{~h}$ cultivation in calcium carbonate (D, E, F) or different MES concentrations (A, B, C), respectively containing screening medium. Error bars indicate the deviation from the mean $(n=2)$.

\section{Conclusions}

Comparative analysis of the mitochondrial and extracellular transporters involved in itaconate and $(S)-2$ hydroxyparaconate biosynthesis by Ustilago maydis and Aspergillus terreus revealed striking differences, which strongly influence the absolute and relative production rates of these two secondary metabolites. This work suggests that fungal production of itaconate and $(S)$-2-hydroxyparaconate involves a complex 
interplay between metabolism, transport, and extracellular $\mathrm{pH}$. It also provides valuable information for the rational design of efficient itaconate and $(S)$-2-hydroxyparaconate producing strains, which was demonstrated by the increased production of $A t \_m t t A$-expressing strains. However, further biochemical characterization of the investigated transporters is needed, and will be the subject of a future study. The At_MfsA transporter is a promising target for the future metabolic engineering of a $(S)$-2-hydroxyparaconate producing strain, since it will help to increase product specificity. In general, this study sheds light on the role of compartmentation, and the associated transporters, of the fungal biosynthesis pathway of itaconate and (S)-2-hydroxyparaconate. It also highlights the relevance of $U$. maydis, both as a model organism, and as an industrial work horse for the production of organic acids.

\section{Conflict of interest}

All authors have seen and approved the manuscript. All authors have contributed significantly to the work. The manuscript has not been published and is not being considered for publication elsewhere. All authors declare that they have no competing financial interests. Used transporters were patented in prior works by Dutch DNA and RWTH-Aachen University.

\section{Acknowledgements}

We thank Kylie L. Luska and Tim den Hartog (Institute for Technical and Macromolecular Chemistry, RWTH-Aachen) for contributions to $(S)$-2-hydroxyparaconate synthesis.

\section{Author's contributions}

All authors have contributed significantly to the work. EG and HHT contributed equally to this work. NW and PP conceived the project. EG and HHT designed and performed experiments and analyzed results with the help of NW, LMB and PP. HHT wrote the manuscript with the help of EG, NW, LMB and PP. SKH performed southern blots, MM constructed $U$. maydis transformants and $\mathrm{AHH}$ provides sequence information and discussion for $A$. terreus transporters.

\section{Funding}

This work was funded by the German Federal Ministry of Food and Agriculture (BMEL), through the Specialist agency renewable raw materials e. V. (FNR) as part of the ERA-IB project "TTRAFFIC". The scientific activities of the Bioeconomy Science Center were supported financially by the Ministry of Innovation, Science and Research within the framework of the NRW Strategieprojekt BioSC (No. 578 313/323-400-002 13). This work was supported by the Excellence Initiative of the German federal state governments as a part of the Cluster of Excellence 'Tailor-Made Fuels from Biomass'.

\section{References}

1. Bambouskova, M., Gorvel, L., Lampropoulou, V., Sergushichev, A., Loginicheva, E., Johnson, K., Korenfeld, D., Mathyer, M.E., Kim, H., Huang, L.H., Duncan, D., Bregman, H., Keskin, A., Santeford, A., Apte, R.S., Sehgal, R., Johnson, B., Amarasinghe, G.K., Soares, M.P., Satoh, T., Akira, S., Hai, T., de Guzman Strong, C., Auclair, K., Roddy, T.P., Biller, S.A., Jovanovic, M., Klechevsky, E., Stewart, K.M., Randolph, G.J., Artyomov, M.N. 2018. Electrophilic properties of itaconate and derivatives regulate the I $\mathrm{B} \zeta$-ATF3 inflammatory axis. Nature 556, 501-504.

2. Bonnarme, P., Gillet, B., Sepulchre, A.M., Role, C., Beloeil, J.C., Ducrocq, C. 1995. Itaconate biosynthesis in Aspergillus terreus. J. Bacteriol. 177, 3573-3578. 
3. Brandt, J.R., Salerno, F., Fuchter, M.J. 2017. The added value of small-molecule chirality in technological applications. Nat. Rev. Chem. 1.

4. Duetz, W.A., Ruedi, L., Hermann, R., O'Connor, K., Buchs, J., Witholt, B. 2000. Methods for intense aeration, growth, storage, and replication of bacterial strains in microtiter plates. Appl. Environ. Microb. 66, 2641-2646.

5. Dwiarti, L., Yamane, K., Yamatani, H., Kahar, P., Okabe, M. 2002. Purification and characterization of cis-aconitic acid decarboxylase from Aspergillus terreus TN484-M1. J. Biosci. Bioeng. 94, 29-33.

6. Geilen, F.M.A., Engendahl, B., Harwardt, A., Marquardt, W., Klankermayer, J., Leitner, W. 2010. Selective and flexible transformation of biomass-derived platform chemicals by a multifunctional catalytic system. Angew. Chem. Int. Edit. 49, 5510-5514.

7. Geiser, E., Przybilla, S.K., Engel, M., Kleineberg, W., Buttner, L., Sarikaya, E., Den Hartog, T., Klankermayer, J., Leitner, W., Bolker, M., Blank, L.M., Wierckx, N. 2016. Genetic and biochemical insights into the itaconate pathway of Ustilago maydis enable enhanced production. Metab. Eng. 38, 427-435.

8. Geiser, E., Przybilla, S.K., Friedrich, A., Buckel, W., Wierckx, N., Blank, L.M., Bolker, M. 2015. Ustilago maydis produces itaconic acid via the unusual intermediate trans-aconitate. Microb. Biotechnol. 9, 116-126.

9. Geiser, E., Wiebach, V., Wierckx, N., Blank, L.M. 2014. Prospecting the biodiversity of the fungal family Ustilaginaceae for the production of value-added chemicals. Fungal Biol. Biotechnol. 1, 2.

10. Guevarra, E.D., Tabuchi, T. 1990a. Accumulation of itaconic, 2-hydroxyparaconic, itatartaric, and malic-acids by strains of the genus Ustilago. Agric. Biol. Chem. 54, 2353-2358.

11. Guevarra, E.D., Tabuchi, T. 1990b. Production of 2-Hydroxyparaconic and itatartaric acids by Ustilago cynodontis and simple recovery process of the acids. Agric. Biol. Chem. 54, 2359-2365.

12. Hewald, S., Josephs, K., Bolker, M. 2005. Genetic analysis of biosurfactant production in Ustilago maydis. Appl. Environ. Microb. 71, 3033-3040.

13. Hossain, A.H., Li, A., Brickwedde, A., Wilms, L., Caspers, M., Overkamp, K., Punt, P.J. 2016. Rewiring a secondary metabolite pathway towards itaconic acid production in Aspergillus niger. Microb. Cell Fact. 15, 130.

14. Huang, X.N., Lu, X.F., Li, Y.M., Li, X., Li, J.J. 2014. Improving itaconic acid production through genetic engineering of an industrial Aspergillus terreus strain. Microb. Cell Fact. 13, 119.

15. Jadwiga, J., Diana, M. 1974. Studies on the metabolic pathway for itartaric acid formation by Aspergillus terreus. Acta Microbiologica Polonica Ser. B 6, 51-61.

16. Jaklitsch, W.M., Kubicek, C.P., Scrutton, M.C. 1991. The Subcellular organization of itaconate biosynthesis in Aspergillus terreus. J. Gen. Microbiol. 137, 533-539.

17. Kanamasa, S., Dwiarti, L., Okabe, M., Park, E.Y. 2008. Cloning and functional characterization of the cis-aconitic acid decarboxylase (CAD) gene from Aspergillus terreus. Appl. Microbiol. Biot. 80, 223-9.

18. Karaffa, L., Diaz, R., Papp, B., Fekete, E., Sandor, E., Kubicek, C.P. 2015. A deficiency of manganese ions in the presence of high sugar concentrations is the critical parameter for achieving high yields of itaconic acid by Aspergillus terreus. Appl. Microbiol. Biot. 99, 7937-44.

19. Klement, T., Buchs, J. 2013. Itaconic acid - A biotechnological process in change. Bioresource. Technol. 135, 422-431.

20. Krull, S., Hevekerl, A., Kuenz, A., Pruss, U. 2017. Process development of itaconic acid production by a natural wild type strain of Aspergillus terreus to reach industrially relevant final titers. Appl. Microbiol. Biot. 101, 4063-4072. 
21. Kuenz, A., Gallenmuller, Y., Willke, T., Vorlop, K.D. 2012. Microbial production of itaconic acid: developing a stable platform for high product concentrations. Appl. Microbiol. Biot. 96, 12091216.

22. Li, A., van Luijk, N., ter Beek, M., Caspers, M., Punt, P., van der Werf, M. 2011. A clone-based transcriptomics approach for the identification of genes relevant for itaconic acid production in Aspergillus. FungaI Genet. Biol. 48, 602-11.

23. Maassen, N., Panakova, M., Wierckx, N., Geiser, E., Zimmermann, M., Bölker, M., Klinner, U., Blank., L.M. 2014. Influence of carbon and nitrogen concentration on itaconic acid production by the smut fungus Ustilago maydis. Eng. Life. Sci. 14, 129-134.

24. Mori, K., Fukamatsu, K. 1992. Pheromone Synthesis .164. A Synthesis of (1r,5s)-(+)-Frontalin from (S)-(-)-2-Hydroxyparaconic Acid. Liebigs Annalen Der Chemie, 1191-1193.

25. Okabe, M., Lies, D., Kanamasa, S., Park, E.Y. 2009. Biotechnological production of itaconic acid and its biosynthesis in Aspergillus terreus. Appl. Microbiol. Biot. 84, 597-606.

26. Przybilla, S.K. 2014. Genetische und biochemische Charakterisierung der Itaconsäure-Biosynthese in Ustilago maydis. in: Biologie, Vol. Dr.rer.nat., Philipps-Universität Marburg.

27. Sarkari, P., Reindl, M., Stock, J., Muller, O., Kahmann, R., Feldbrugge, M., Schipper, K. 2014. Improved expression of single-chain antibodies in Ustilago maydis. J. Biotechnol 191, 165-175.

28. Schulz, B., Banuett, F., Dahl, M., Schlesinger, R., Schafer, W., Martin, T., Herskowitz, I., Kahmann, R. 1990. The b-Alleles of $U$. maydis, whose combinations program pathogenic development, code for polypeptides containing a homeodomain-related motif. Cell 60, 295-306.

29. Spellig, T., Bottin, A., Kahmann, R. 1996. Green fluorescent protein (GFP) as a new vital marker in the phytopathogenic fungus Ustilago maydis. Molecular \& General Genetics 252, 503-509.

30. Steiger, M.G., Blumhoff, M.L., Mattanovich, D., Sauer, M. 2013. Biochemistry of microbial itaconic acid production. Front. Microbiol. 4.

31. Steiger, M.G., Punt, P.J., Ram, A.F.J., Mattanovich, D., Sauer, M. 2016. Characterizing MttA as a mitochondrial cis-aconitic acid transporter by metabolic engineering. Metab. Eng. 35, 95-104.

32. Stodola, F.H., Friedkin, M., Moyer, A.J., Coghill, R.D. 1945. Itatartaric acid, a metabolic product of an ultraviolet-induced mutant of Aspergillus terreus. J. Biol. Chem. 161, 739-742.

33. van der Straat, L., Vernooij, M., Lammers, M., van den Berg, W., Schonewille, T., Cordewener, J., van der Meer, I., Koops, A., de Graaff, L.H. 2014. Expression of the Aspergillus terreus itaconic acid biosynthesis cluster in Aspergillus niger. Microb. Cell Fact. 13.

34. Weastra. 2014. S.R.O., 2013. In: Weastra, S.R.O. (ed.), Determination of market potential for selected platform chemicals WP 8.1 Slovakia.

35. Werpy, T., Peterson, G. 2004. Top value added chemicals from biomass. 2004 US-DoE report PNNL16983.

36. Willis, R.B., Montgomery, M.E., Allen, P.R. 1996. Improved Method for Manual, Colorimetric Determination of Total Kjeldahl Nitrogen Using Salicylate. J. Agr. Food Chem. 44, 1804-1807.

37. Willke, T., Vorlop, K.D. 2001. Biotechnological production of itaconic acid. Appl. Microbiol. Biot. 56, 289-295.

38. Zambanini, T., Hartmann, S.K., Schmitz, L.M., Büttner, L., Hosseinpour Tehrani, H., Geiser, E., Beudels, M., Venc, D., Wandrey, G., Büchs, J., Schwarzländer, M., Blank, L.M., Wierckx, N. 2017a. Promoters from the itaconate cluster of Ustilago maydis are induced by nitrogen depletion. Fungal Biol. Biotechnol. 4, 11. 
39. Zambanini, T., Hosseinpour Tehrani, H., Geiser, E., Sonntag, C.K., Buesche, J.M.r., Meurer, G., Nick, W., Blank, L.M. 2017b. Metabolic engineering of Ustilago trichophora TZ1 for improved malic acid production. Metab. Eng. Commun. 4, 12-21.

40. Zarnack, K., Maurer, S., Kaffarnik, F., Ladendorf, O., Brachmann, A., Kamper, J., Feldbrugge, M. 2006. Tetracycline-regulated gene expression in the pathogen Ustilago maydis. Fungal Genetics and Biology 43, 727-738. 
Supplemental data to:

The interplay between transport and metabolism in fungal itaconic acid production

Hamed Hosseinpour Tehrani ${ }^{1 \ddagger}$ Elena Geiser ${ }^{1,4 \ddagger}$, Meike Engel ${ }^{1}$, Sandra K. Hartmann ${ }^{1}$, Abeer H. Hossain ${ }^{2}$,

Peter J. Punt ${ }^{2}$, Lars M Blank ${ }^{1} \&$ Nick Wierckx*1,3

$\$$ These authors contributed equally to this manuscript

*to whom correspondence should be addressed

${ }^{1}$ iAMB - Institute of Applied Microbiology, ABBt - Aachen Biology and Biotechnology, RWTH Aachen

University, Worringerweg 1, 52074 Aachen, Germany

${ }^{2}$ Dutch DNA Biotech B.V, Utrechtseweg 48, 3704 HE, Zeist, The Netherlands

${ }^{3}$ Institute of Bio- and Geosciences IBG-1: Biotechnology, Forschungszentrum Jülich, 52425 Jülich,

Germany

${ }^{4}$ BioSC, c/o Forschungszentrum Jülich, 52425 Jülich, Germany

Address for correspondence:

Prof. Dr. Nick Wierckx

Phone: $\quad$ +4924616185247

Fax: $\quad$ +492461612710

email: $\quad$ n.wierckx@fz-juelich.de 


\section{Supplemental data}

D1. Annotated sequence of $m t t A$ dicodon-optimized for U. maydis.

\section{BamHI \\ -+----}

1 cactataggg cgaattgaag gaaggccgtc aaggecgcat gtcggatcca tgggtcacgg dicodon-optimized MttA >>........

$\mathrm{m} \mathrm{g} \mathrm{h}$

61 cgacaccgag tcgcccaacc ccacgaccac caccgaggge tcgggtcaaa acgagcccga

$>$...................dicodon-optimized MttA

g d t e s p n p t t t t e g s g q n e p

121 gaagaagggt cgtgacatcc cgctctggeg caagtgcgtc atcacetttg tcgtctcgtg

$>$...................dicodon-optimized MttA..................... >

e k k g r d i p l w r k c v i t f v v s

181 gatgacgctc gtggtcactt tctcgtcgac ctgtctgctg cecgccgctc ccgagatcgc $>$....................dicodon-optimized MttA..................... >

w m t l v v t f s s t c ll pa a p e i

241 caacgagttt gacatgaccg tcgagaccat caacatctcg aacgccggtg tcctcgtcgc

$>$...................dicodon-optimized MttA

a $n$ e f d m t ve t i n is n a g v l v

301 catgggetac tcgtcgctta tctggggtcc catgaacaag ctcgttggec gtcgcacctc

$>$....................dicodon-optimized MttA

a m g y s s l i w g p m n k l v g r r t

361 gtacaacctc gctatctcga tgctctgcge ttgctcggcg ggcacggccg ctgccatcaa

$>$....................dicodon-optimized MttA......................>

s y n l a i s m l c a c s a g t a a a i

421 cgaggagatg ttcatcgcet tccgcgtgct ctccggtctg accggtacct cgttcatggt

$>$.....................dicodon-optimized MttA..................... >

n e e m f i a f r v l s g l t g t s f m

481 ctcgggtcag acggtgctcg ccgacatctt cgagccegtc taccgeggca ccgccgtcgg

$>$....................dicodon-optimized MttA..................... >

v s g q t v l a d i f e p v y r g t a v

541 cttcttcatg gecggtaccc tctcgggtcc cgctatcggt ccgtgcgtcg gtggtgtcat

$>$...................dicodon-optimized MttA................... >

g f f m a g t l s g paig p c v g g v

601 cgtcacettc acctcgtgge gtgtcatctt ctggetccag ctcggcatgt cgggtctcgg

$>$....................dicodon-optimized MttA..................... >

i v t f t s s w r v i f w 1 q 1 g m s 
661 tctcgtcctc tcgctcctct tcttccccaa gatcgagggc aactcggaaa aggtctcgac $>$...................dicodon-optimized MttA..................... g 1 v l s l l f f p k i e g n s e k v s

721 cgcettcaag cccaccacgc tcgtcaccat catcagcaag ttctcgecca ccgacgtgct $>$...................dicodon-optimized MttA.....................

$\mathrm{t}$ a f k p t t l v t i i s k f s p t d v

781 caagcagtgg gtctacccea acgtcttcct cgetgacetc tgetgtggtc tgctcgecat

$>$...................dicodon-optimized MttA....................>

$1 \mathrm{k} \mathrm{q}$ w $\mathrm{v}$ y $\mathrm{p}$ n v f 1 a d l c c g 11 a

841 cacccagtac tcgatcetca cetcggegeg tgccatcttc aactcgegct tccacctcac

$>$....................dicodon-optimized MttA..................... >

i t q y s i l t s a r a i f $\mathrm{ns} r \mathrm{f} \mathrm{h} \mathrm{l}$

901 cactgctctc gtctctggtc tettctacet cgctcceggt gecggettcc tcatcggctc

$>$....................dicodon-optimized MttA..................... >

t t a 1 v s g 1 f y 1 a p g a g f 1 i g

961 gctcgttggt ggcaagctct cggaccgcac cgtccgecgc tacatcgtca agcgcggctt $>$....................dicodon-optimized MttA..................... >

s $1 \mathrm{v}$ g $\mathrm{g}$ k 1 s d r t v r r y i v $\mathrm{k}$ r g

1021 ccgtctgecg caggaccgtc tccactcggg tctgatcacg etgttcgecg tectccecgc $>$....................dicodon-optimized MttA..................... >

f r l p q d r l h s g 1 i t 1 f a v l p

1081 cggtacgctc atctacgget ggacgctcca ggaggacaag ggcgacatgg tggtgcccat $>$....................dicodon-optimized MttA......................

a g t 1 i y g w t 1 q e d k g d m v v p

1141 catcgecgec ttcttcgccg getggggtct catgggctcg ttcaactgcc tcaacacgta $>$....................dicodon-optimized MttA .>

i i a f f a g w g $1 \mathrm{mg}$ s f n c $1 \mathrm{n} \mathrm{t}$

1201 cgtcgccgag gegctgccgc gcaaccgttc cgccgtcatt gecggcaagt acatgatcca $>$...................dicodon-optimized MttA..................... > y v a e a 1 p r n r s a vi a g k y m i

1261 gtacaccttt tcggccgget cgtcggeget cgtcgtccce gtcatcgatg cgctcggcgt $>$...................dicodon-optimized MttA................... >

q y t f s a g s s a 1 v v p vid a $1 \mathrm{~g}$

1321 cggcgagacc ttcacgetct gegtcgtcgc ctcgaccatc gecggtctca tcaccgccgc

$>$....................dicodon-optimized MttA..................... >

v g e t f t l c v v a s t i a g l i t a

1381 catcgctcgc tggggtatca acatgcagcg atgggccgag cgtgccttca acatgccac $>$....................dicodon-optimized MttA

a $\mathrm{i}$ a $\mathrm{r}$ w $\mathrm{g}$ n m q $\mathrm{r}$ w a e $\mathrm{r}$ a $\mathrm{n} \mathrm{m} \mathrm{p}$ 
NotI

--+------

1441 ccagtgagcg gecgccegct gggectcatg ggcettcctt tcactgcceg ctttccag

$>$....> dicodon-optimized MttA

t q -

D2. Annotated sequence of $m f_{s} A$ dicodon-optimized for $U$. maydis.

\section{BamHI}

-+----

1 cgaattgaag gaaggccgtc aaggccacgt gtcttgtcca ggcgegccag tcggatccat dicodon-optimized MfsA >>

61 ggactcgaag atccagacca acgtgccgct ccccaaggct ccgctcatcc agaaggcgeg $>$...................dicodon-optimized MfsA.....................

m d s k i q t n v p l p k a pli q k a

121 cggcaagcgc accaagggca tccetgcget ggtcgctggt gectgcgecg gtgccgtcga $>$....................dicodon-optimized MfsA.....................

r g k r t k g i p a l v a g a c a g a v

181 gatctcgatc acctaccect tcgagtcggc caagaccegt getcagctca agcgtcgcaa $>$....................dicodon-optimized MfsA.....................

e i s i t y p f e s a k t r a q l k r r

241 ccacgacgtc gecgecatca agcceggtat ccgeggetgg tacgecggct acggtgccac $>$....................dicodon-optimized MfsA.....................

n h d v a a i k p g i r g w y a g y g a

301 gctcgtcggc accaccgtca aggcgtcggt gcagttcgec tcgttcaaca tctaccgatc $>$.....................dicodon-optimized MfsA...................... >

t 1 v g t t v k a s v q f a s f n i y r

361 ggcgetctcg ggtccaacg gtgagctctc gaccggtgcc tcggtgctcg ccggctttgg

$>$....................dicodon-optimized Mfs A..................... >

$\mathrm{s}$ a $1 \mathrm{~s}$ g p n g e l s t g a s v l a $\mathrm{g} \mathrm{f}$

421 tgccggtgtc accgaggcgg tcctcgecgt cacgcccgec gaggccatca aaaccaagat $>$...................dicodon-optimized MfsA.................... >

g a g v t e a v l a v t p a e a i k t k

481 catcgatgct cgcaaggtcg gcaacgccga getgtcgacc accttcggtg ccatcgccgg

$>$....................dicodon-optimized MfsA.................... >

i i d a r k g g a e l s t t f g a i a

541 catcetgege gaccgtggtc cgctcggctt cttctcggec gtcggtccca ccatcetgeg 
g i 1 r d r g p l g f f s a v g p t i 1

601 tcagtcgtcc aacgecgecg tcaagttcac cgtctacaac gagctcatcg gtctcgctcg

$>$....................dicodon-optimized MfsA..................... >

r q s s n a a v k f t v y n e 1 i g 1 a

661 taagtacagc aagaacggcg aggacgtcca cccgctcgec tcgacgctgg tcggctcggt

$>$.....................dicodon-optimized MfsA..................... >

$\mathrm{r}$ k y s k n g e d v h p la s t l v g s

721 caccggtgtc tgctgtgect ggtcgacgca gccgctcgac gtcatcaaga cgcgcatgca

$>$....................dicodon-optimized MfsA.................... >

v t g v c c a w s t q p l d v i k t r m

781 gtcgctccag gcgcgtcagc tctacggcaa cacgttcaac tgcgtcaaga cgctgctgcg

$>$....................dicodon-optimized MfsA....................>>

q s l q a r q 1 y g $\mathrm{g}$ t f $\mathrm{n}$ c v $\mathrm{k}$ t $1 \mathrm{ll}$

841 ctccgagggc atcggtgtct tctggtcggg tgtctggttc cgcaccggtc gtctctcgct

$>$...................dicodon-optimized MfsA....................

r s e g i g v f w s g v w f r t g r l s

901 cacctcggec atcatgttcc ccgtctacga gaaggtctac aagttcctca cccagcccaa $>$....................dicodon-optimized MfsA....................>

$1 \mathrm{t} s$ a $\mathrm{i} m \mathrm{f} p \mathrm{v}$ y e k v y $\mathrm{k} f \mathrm{l}$ t $\mathrm{p}$

\section{NotI}

--+------

961 ctgagcggec geccgattaa ttaatggagc acaagactgg cetcatggge cttcetttca >.>> dicodon-optimized MfsA

$\mathrm{n}-$

$1021 \mathrm{ctgc}$ 


\section{Supplemental figures}

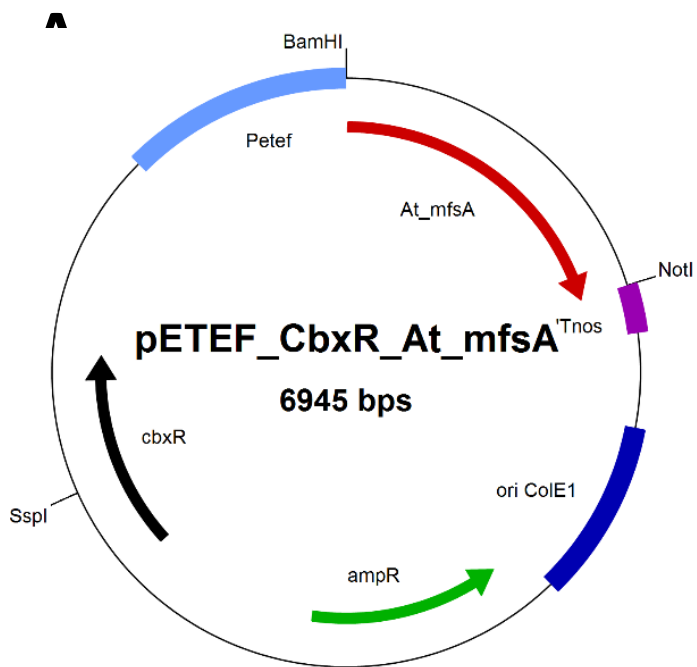

Plasmid maps of pETEF_CbxR_At_mttA and

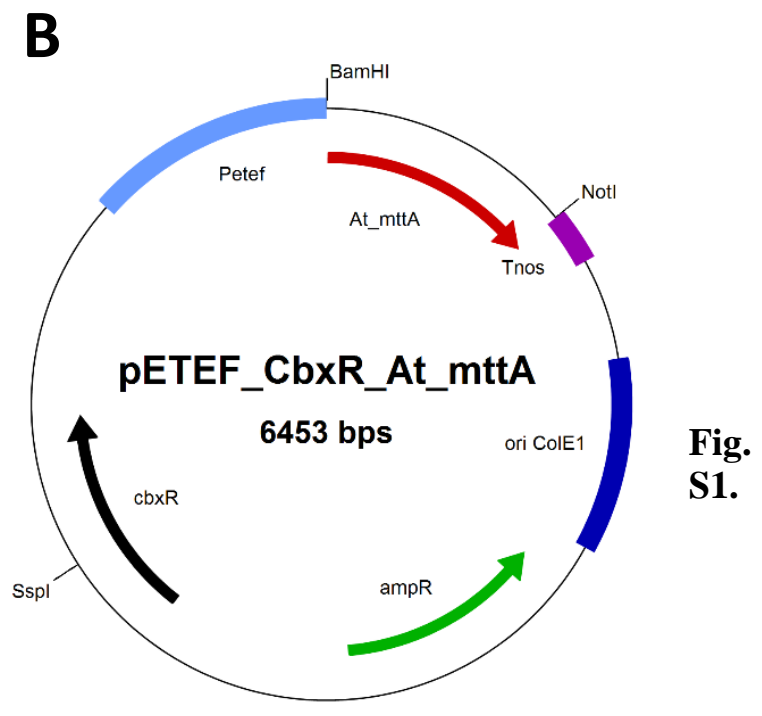
pETEF_CbxR_At_mfsA. Both plasmids contain, cbxR: carboxin resistance, ampR: ampicillin resistance, ori ColE1: origin of replication E. coli, $\mathrm{P}_{\text {etef: }}$ constitutive promoter, SspI, BamHI and NotI as restriction sites, A: dicodon-optimized $m f s A$ and B: dicodon-optimized $m t t A$. 